\title{
Precise Measurement of Electron Antineutrinos Disappearance From the Daya Bay Experiment
}

\author{
Rupert Leitner* \\ for the Daya Bay Collaboration ${ }^{\dagger}$ \\ Charles University, Prague, Czech Republic \\ E-mail: Rupert.Leitner@mff.cuni.cz
}

In March 2012 the Daya Bay reactor neutrino experiment observed the disappearance of electron antineutrinos at about $2 \mathrm{~km}$. Within the three-neutrino flavor framework this result can be used for measuring a value of $\sin ^{2}\left(2 \theta_{13}\right)$ for the least known neutrino mixing angle $\theta_{13}$. The exposure of $116.8 \mathrm{kton}-\mathrm{GW}_{t h}$-day improved the result to $\sin ^{2} 2 \theta_{13}=0.089 \pm 0.010_{\text {stat }} \pm 0.005_{\text {syst }}$, the most precise measurement of $\theta_{13}$ to date. A short overview of the experiment, results and prospects are presented.

The European Physical Society Conference on High Energy Physics -EPS-HEP2013

18-24 July 2013

Stockholm, Sweden

\footnotetext{
*Speaker.

${ }^{\dagger}$ Daya Bay is supported in part by the Ministry of Science and Technology of China, the United States Department of Energy, the Chinese Academy of Sciences, the National Natural Science Foundation of China, the Guangdong provincial government, the Shenzhen municipal government, the China Guangdong Nuclear Power Group, Shanghai Laboratory for Particle Physics and Cosmology, the Research Grants Council of the Hong Kong Special Administrative Region of China, University Development Fund of The University of Hong Kong, the MOE program for Research of Excellence at National Taiwan University, National Chiao-Tung University, and NSC fund support from Taiwan, the U.S. National Science Foundation, the Alfred P. Sloan Foundation, the Ministry of Education, Youth and Sports of the Czech Republic, Charles University in Prague, the Joint Institute of Nuclear Research in Dubna, Russia, and the CNFC-RFBR joint research program. We acknowledge Yellow River Engineering Consulting Co., Ltd. and China railway 15th Bureau Group Co., Ltd. for building the underground laboratory. We are grateful for the ongoing cooperation from the China Guangdong Nuclear Power Group and China Light \& Power Company.
} 


\section{Neutrino mixing and oscillations}

Neutrino flavor eigenstates $v_{f}$ originating in weak decays together with charged leptons of three known flavors $f=e, \mu, \tau$ are super-positions of three mass eigenstates $v_{i} i=1,2,3$ with masses $m_{1}, m_{2}, m_{3}$. The experimentally firmly established phenomenon of oscillations of neutrino flavors can be explained by a non-diagonal mixing matrix and different masses of neutrinos mass eigenstates. The canonical representation of the mixing matrix is the ordered product of three rotations with angles $\theta_{12}, \theta_{13}, \theta_{23}$ and one CP violating phase $\delta$. In the case of a Majorana origin of the neutrino the mixing matrix contains another two so called Majorana phases. Oscillations of neutrino flavors do not depend on Majorana phases and the part of the mixing matrix relevant for oscillations is:

$$
U_{f i}=\left(\begin{array}{ccc}
1 & 0 & 0 \\
0 & c_{23} & s_{23} \\
0 & -s_{23} & c_{23}
\end{array}\right)\left(\begin{array}{ccc}
c_{13} & 0 & s_{13} e^{i \delta} \\
0 & 1 & 0 \\
-s_{13} e^{-i \delta} & 0 & c_{13}
\end{array}\right)\left(\begin{array}{ccc}
c_{12} & s_{12} & 0 \\
-s_{12} & c_{12} & 0 \\
0 & 0 & 1
\end{array}\right)
$$

where $s_{i j}$ and $c_{i j}$ denote $\sin \theta_{i j}$ and $\cos \theta_{i j}$ respectively.

With an approximation of $\Delta m_{31}^{2} \equiv m_{3}^{2}-m_{1}^{2} \cong m_{3}^{2}-m_{2}^{2} \equiv \Delta m_{32}^{2}$ the survival probability for electron antineutrino with energy $E$ at the distance $x$ from the source is given by the formula:

$$
P_{\bar{v}_{e} \rightarrow \bar{v}_{e}}\left(\frac{x}{E}\right)=1-\sin ^{2}\left(2 \theta_{13}\right) \sin ^{2}\left(\frac{\Delta m_{31}^{2} x}{4 \hbar c E}\right)-\cos ^{4}\left(\theta_{13}\right) \sin ^{2}\left(2 \theta_{12}\right) \sin ^{2}\left(\frac{\Delta m_{21}^{2} x}{4 \hbar c E}\right)
$$

The Daya Bay experiment [1] was designed to measure the value of the least known oscillation parameter $\theta_{13}$ with unprecedented precision by employing a relative rate measurement of electron antineutrinos between a far site and two near sites located at a nuclear power plant in south China.

First hints of the non-zero mixing angle $\theta_{13}$ arose from combinations of solar neutrino data and KamLAND's reactor neutrino data [2]. It was followed by indications in accelerator neutrino measurements by T2K [3] and MINOS [4], and reactor neutrino experiment Double-CHOOZ [5] with data departing from $\theta_{13}=0$ hypothesis at 1.7 to $2.5 \sigma$ level. With the first six 20-ton targetmass detectors of identical design the Daya Bay Experiment [6] reported observation of non-zero mixing angle $\theta_{13}$ with significance at $5.2 \sigma$ in March 2012. The measurement was subsequently confirmed by RENO [7]. In June 2012 the Daya Bay experiment published improved result with increased statistics [8], the most precise measurement to date.

\section{Detection of reactor antineutrinos}

Nuclear power reactors are very powerful sources of electron antineutrinos. Fission products are neutron rich isotopes that are transformed to stable nuclei via a series of $\beta^{-}$decays. On average $\approx 6$ electron antineutrinos follow each fission producing a flux of $\approx 2 \cdot 10^{20}$ of $\bar{v}_{e}$ per second per $1 \mathrm{GW}_{t h}$ of reactor thermal power. The energy spectrum of antineutrinos decreases with the energy and extends up to $\approx 10 \mathrm{MeV}$. Antineutrinos with energies $E_{v} \geq 1.8 \mathrm{MeV}$ can be detected by charged current interactions (so called inverse beta decay IBD):

$$
\bar{v}_{e}+p \rightarrow e^{+}+n
$$


on target protons of liquid scintillator. The detected antineutrinos energy spectrum is the product of the original antineutrino flux and the neutrino energy dependent IBD cross section. The detected spectrum has a typical shape with a maximum at $E_{v} \approx 4 \mathrm{MeV}$.

The positron losses its kinetic energy by ionization and annihilates with an electron producing a pair of $m_{e}=511 \mathrm{keV}$ gammas. This so called prompt signal $E_{p}$ is related to the neutrino energy $E_{v} \cong E_{p}+0.8 \mathrm{MeV}$.

The kinetic energy of IBD neutrons is $\approx 10 \mathrm{keV}$. Neutrons are thermalized by elastic collisions with nuclei of liquid scintillator. In order to separate the neutron signal from backgrounds, liquid scintillator is doped with Gadolinium. The two isotopes ${ }^{155} \mathrm{Gd}$ and ${ }^{157} \mathrm{Gd}$ have huge cross sections for thermal neutrons capture (61 and $254 \mathrm{k}$ barns). Neutron capture on these Gd isotopes is followed by the emission of several gammas with total energies of $8.54 \mathrm{MeV}$ and $7.94 \mathrm{MeV}$ respectively. As the abundances of ${ }^{155} \mathrm{Gd}$ and ${ }^{157} \mathrm{Gd}$ are high $(14.8 \%$ and $15.7 \%)$ the addition of the small amount of $0.1 \%$ of $\mathrm{Gd}$ is enough to ensure a capture of more than $80 \%$ of neutrons on Gd. The time difference $\Delta t$ between the prompt signal from positron and the delayed signal from the neutron follows an exponential distribution $e^{-\Delta t /\langle\tau\rangle}$ with the value of capture time $\langle\tau\rangle \cong 29 \mu \mathrm{s}$ that is inversely proportional to Gd concentration.

The number of detected IBD events is proportional to the product of the detector mass and power of the nuclear reactors. In addition the optimal sensitivity requires placing the far detector(s) at a distance close to $2 \mathrm{~km}$ where the maximal disappearance is expected for neutrinos around the peak energy of $4 \mathrm{MeV}$. Correlated systematic errors are cancelled and uncorrelated reactor uncertainties are minimized in a configuration with identical near detector(s) [9] placed closer to the reactors where oscillations effects are small. The background can be minimized by a large overburden. The near-far arrangement of the Daya Bay antineutrino detectors (ADs) is shown in Fig. 1 and relevant parameters are summarized in the following table:

\begin{tabular}{|c|c|cc|cc|cc|}
\hline & Reactor Power & \multicolumn{2}{|c|}{ Detector mass [t] } & \multicolumn{2}{|c|}{ Baseline $[\mathrm{m}]$} & \multicolumn{2}{|c|}{ Overburden [mwe] } \\
& {$\left[\mathrm{GW}_{t h}\right]$} & Near & Far & Near & Far & Near & Far \\
\hline & 17.4 & 40,40 & 80 & 498,555 & 1628 & 250,265 & 860 \\
\hline
\end{tabular}

Each Daya Bay antineutrino detector [10] is a stainless steel vessel with three nested cylindrical volumes separated by two concentric acrylic vessels. The innermost volume serves as the target and is filled with $20 \mathrm{t}$ of Gd loaded liquid scintillator (Gd-LS). In order to fully contain gammas from positron annihilation and $\mathrm{Gd}$ de-excitation the central volume is surrounded by a gamma catcher of 21t of Gd free liquid scintillator (LS) in an outer acrylic vessel. The outermost volume of $37 \mathrm{t}$ of mineral oil serves as shielding of the inner volumes from radiation originating from the walls of stainless steel vessel and photomultipliers. To increase the light yield and improve the homogeneity reflector panels are installed at the top and bottom of the outer acrylic vessel. Scintillating light is detected by 192 8-inch PMTs placed along the circumference of the stainless steel vessel. The Daya Bay detectors are submerged in instrumented water pools such that at least $2.5 \mathrm{~m}$ of water is surrounding each detector. Purified water passively shields detectors from the ambient and cosmic rays induced radioactivity and actively detects Cherenkov light produced by cosmic muons. In addition, an array of resistive plate chamber (RPC) modules is placed on top of the pools for increased background rejection. 
The detectors are equipped with three automated calibration units ACU A, B and C that insert calibration sources along the vertical direction in the centre of the detector, close to the edge of target volume and in the middle of gamma catcher. The calibration near the IBD threshold is done with ${ }^{68} \mathrm{Ge}$ that decays by electron capture to $\beta^{+}$emitter ${ }^{68} \mathrm{Ga}$. Near the peak of the detected antineutrino energy spectrum the detectors are calibrated with ${ }^{60} \mathrm{Co}$ radioactive source. Neutrons from ${ }^{241} \mathrm{Am}-{ }^{13} \mathrm{C}$ source are used to calibrate the detector's response to gammas from neutron capture on $\mathrm{H}$ and $\mathrm{Gd}$. The PMT timing, gain and relative quantum efficiencies are calibrated using Light-Emitting Diodes (LED) light. The detector's response is also calibrated by neutrons produced by cosmic rays. The energy resolution of the Daya Bay detectors has been measured to satisfy the relation $\sigma / E=7.5 \% / \sqrt{E[\mathrm{MeV}]}+0.9 \%$. The value of the statistical terms of $7.5 \%$ is in good agreement with the measured yield of 160 photoelectrons per MeV.

\subsection{IBD candidates selection}

The minimum value of the IBD signal at the threshold is $1.022 \mathrm{MeV}$. Due to energy resolution and possible inhomogeneity of the response, the prompt signal is selected in the region 0.7 $\mathrm{MeV}<E_{p}<12 \mathrm{MeV}$. The delayed signal $6 \mathrm{MeV}<E_{d}<12 \mathrm{MeV}$ from the neutron capture on $\mathrm{Gd}$ is searched in the time window $\Delta t \equiv t_{d}-t_{p}=1-200 \mu$ s following the prompt signal. In addition there should be no signal exceeding $0.7 \mathrm{MeV}$ in $200 \mu$ s time windows preceding and following the prompt signal and the delayed signal respectively (so called multiplicity cut).

In order to suppress the background the Daya Bay detectors are vetoed for $0.6 \mathrm{~ms}, 1 \mathrm{~ms}$ and $1 \mathrm{~s}$ after so called pool muon, non-showering AD muon and showering $\mathrm{AD}$ muon signals respectively.

\subsection{Systematic uncertainties and Background}

Dominant contributions to detector related uncorrelated uncertainties are uncertainties of the delayed energy cut efficiency and of the fraction of neutron captures on Gd. Influences of uncorrelated reactor based uncertainties to the measured ratio of far to near IBD rates are highly reduced.

The signal of IBD events can be mimic by three major types of background events.

Accidental background are coincidences of prompt and neutron-like signals not caused by IBD events. This background is estimated using data and it is concentrated at low energies.

Fast neutrons background: Energetic neutrons created in photonuclear interactions of cosmic muons could mimic IBD by recoiling off a proton before being captured on Gd. The number of background events can be estimated by the extrapolation of the background shape at energies above $12 \mathrm{MeV}$.

Very neutron rich exotic nuclei created in interactions of cosmic muons. These are mostly ${ }_{3}^{9} \mathrm{Li}$ and less frequently also ${ }_{2}^{8} \mathrm{He}$. A large fraction of ${ }_{3}^{9} \mathrm{Li}$ and ${ }_{2}^{8} \mathrm{He} \beta^{-}$decays are accompanied by a free neutron:

$$
\begin{array}{cc}
{ }_{3}^{9} \mathrm{Li} \rightarrow 2 \alpha+n+e^{-}+\bar{v}_{e} & Q=13.607 \mathrm{MeV} \\
{ }_{2}^{8} \mathrm{He} \rightarrow{ }_{3}^{7} \mathrm{Li}+n+e^{-}+\bar{v}_{e} & Q=10.651 \mathrm{MeV}
\end{array}
$$

Due to the large $\mathrm{Q}$ value $\beta^{-}$-n decays of these nuclei mimic IBD candidates spanning the whole spectrum and because of their long half-lives of 178 and $119 \mathrm{~ms}$ detectors must be vetoed for long times after showering cosmic muon signals.

Daily rates of the most significant backgrounds are summarized in Table 1. 


\begin{tabular}{|ccccccc|}
\hline rates per day & $\mathrm{AD} 1$ & $\mathrm{AD} 2$ & $\mathrm{AD} 3$ & $\mathrm{AD} 4$ & $\mathrm{AD}$ & $\mathrm{AD}$ \\
\hline accidentals & $9.73 \pm 0.10$ & $9.61 \pm 0.10$ & $7.55 \pm 0.08$ & $3.05 \pm 0.04$ & $3.04 \pm 0.04$ & $2.93 \pm 0.03$ \\
fast-neutron & $0.77 \pm 0.24$ & $0.77 \pm 0.24$ & $0.58 \pm 0.33$ & $0.05 \pm 0.02$ & $0.05 \pm 0.02$ & $0.05 \pm 0.02$ \\
${ }^{9} \mathrm{Li} /{ }^{8} \mathrm{He}($ per AD) & \multicolumn{2}{c}{$2.9 \pm 1.5$} & $2.0 \pm 1.1$ & & $0.22 \pm 0.12$ & \\
$\mathrm{Am}-\mathrm{C}$ (per AD) & \multicolumn{7}{c}{$0.2 \pm 0.2$} \\
${ }^{13} \mathrm{C}(\alpha, \mathrm{n}){ }^{16} \mathrm{O}$ & $0.08 \pm 0.04$ & $0.07 \pm 0.04$ & $0.05 \pm 0.03$ & $0.04 \pm 0.02$ & $0.04 \pm 0.02$ & $0.04 \pm 0.02$ \\
\hline $\mathrm{IBD}$ rate & $662.47 \pm 3.00$ & $670.87 \pm 3.01$ & $613.53 \pm 2.69$ & $77.57 \pm 0.85$ & $76.62 \pm 0.85$ & $74.97 \pm 0.84$ \\
\hline
\end{tabular}

Table 1: Summary of significant contributors to the background of the $\bar{v}_{e}$ signal. Daily rates of the backgrounds as well as the signal are shown. The uncertainty of the Am-C background was assumed to be fully correlated among the detectors.

\section{Results}

The $\bar{v}_{e}$ rate in the far hall was predicted with a weighted combination of the two near hall measurements assuming no oscillation. A ratio of the measured to expected rate is defined as

$$
R=\frac{M_{f}}{\bar{N}_{f}}=\frac{M_{f}}{\alpha M_{a}+\beta M_{b}},
$$

where $\bar{N}_{f}$ and $M_{f}$ are the predicted and measured rates in the far hall (sum of AD 4-6), $M_{a}$ and $M_{b}$ are the measured, background-subtracted IBD rates in detectors placed in EH1 and EH2, respectively.
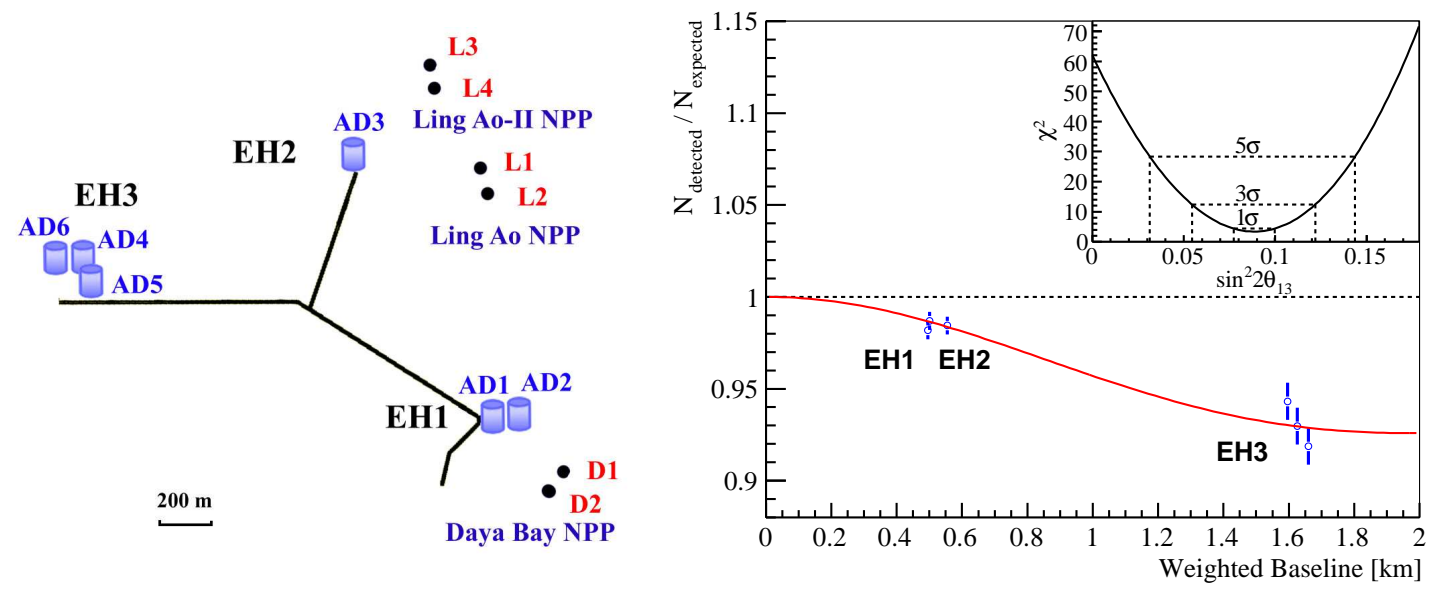

Figure 1: Left: Layout of the Daya Bay experiment. The dots represent reactor cores, labelled as D1, D2, L1, L2, L3 and L4. Six antineutrino detectors (ADs) were installed in three experimental halls (EHs).

Right:Ratio of measured versus expected signals in each detector hall, assuming no oscillation. The oscillation survival probability at the best-fit value is given by the smooth curve. The data points of two ADs in the far hall were displaced by -30 and $+30 \mathrm{~m}$ for visual clarity. The $\chi^{2}$ value versus $\sin ^{2} 2 \theta_{13}$ is shown in the inset.[8]. 
The values for weights $\alpha$ and $\beta$ were dominated by the baselines, and only slightly dependant on the integrated flux of each core. The ratio observed at the far hall was: $R=0.944 \pm$ 0.007 (stat.) \pm 0.003 (syst.) where the statistical (systematic) uncertainties were obtained by propagating statistical (uncorrelated systematic) uncertainties in the measured IBD counts in the three halls. The value of $\sin ^{2} 2 \theta_{13}$ was determined by minimizing a $\chi^{2}$ functional constructed with pull terms accounting for the correlation of the systematic errors, see details in [8]. The best-fit value is $\sin ^{2} 2 \theta_{13}=0.089 \pm 0.010$ (stat.) \pm 0.005 (syst.) with a $\chi^{2} / \mathrm{NDF}$ of $3.4 / 4$. All best estimates of pull parameters are within one standard deviation based on the corresponding systematic uncertainties. The no-oscillation hypothesis is excluded at 7.7 standard deviations.

\section{Conclusion and Outlook}

A non zero, surprisingly large value of the third mixing angle $\theta_{13}$ has been measured by Daya Bay experiment. The presented result $\sin ^{2} 2 \theta_{13}=0.089 \pm 0.010$ (stat.) \pm 0.005 (syst.) is the most precise measurement of the mixing angle $\theta_{13}$ to date. The result is extremely important as it is opening future searches for violation of CP symmetry.

On October 19, 2012, the experiment started taking data in the full configuration with 8 detectors placed in the three experimental halls. With 3 years of data taking the Daya Bay experiment will measure the value of $\sin ^{2} 2 \theta_{13}$ with $\sim 5 \%$ precision.

Performing the spectral analysis, the Daya Bay experiment will be able to measure the position of the oscillation minimum (the effective mass splitting $\Delta m_{e e}^{2}$ ) with precision better than $5 \%$.

With highest statistics collected, Daya Bay will also give precise measurement of the energy spectrum of the reactor antineutrinos. Such measurement is valuable not only for the future reactor neutrino experiments but also for investigation of possible new physics beyond 3-neutrino oscillation.

\section{References}

[1] X. Guo et al.[Daya Bay Collaboration], BNL-77369-2006-IR, LBNL-62137, TUHEP-EX-06-003, Jan 2007. $156 \mathrm{pp}$

[2] G. Fogli et al., Phys. Rev. Lett. 101, 141801 (2008).

[3] K. Abe et al. [T2K Collaboration], Phys. Rev. Lett. 107, 041801 (2011).

[4] P. Adamson et al. [MINOS], Phys. Rev. Lett. 107, 181802 (2011).

[5] Y. Abe et al. (Double Chooz Collaboration), Phys. Rev. Lett. 108, 131801 (2012).

[6] F. P. An et al. [Daya Bay Collaboration], Phys. Rev. Lett. 108, 171803 (2012).

[7] J.K. Ahn et al. (RENO Collaboration), Phys. Rev. Lett. 108, 191802 (2012).

[8] F. P. An et al. [Daya Bay Collaboration], Chinese Physics C37, 011001 (2013), arXiv:1210.6327 [hep-ex].

[9] L.Mikaelyan and V.V.Sinev, Phys.Atom.Nucl.63:1002-1006,2000; Yad.Fiz.63 N6:1077-1081,2000

[10] F. P. An et al. [Daya Bay Collaboration], Nucl. Instr. and Meth. A 685, 78 (2012). 